\title{
Auditory Temporal Order Discrimination and Backward Recognition Masking in Adults With Dyslexia
}

\section{Yvonne M. Griffiths Nicholas I. Hill University of Essex, Colchester, Essex, United Kingdom \\ Peter J. Bailey Margaret J. Snowling University of York, Heslington, York, United Kingdom}

The ability of 20 adult dyslexic readers to extract frequency information from successive tone pairs was compared with that of IQ-matched controls using temporal order discrimination and auditory backward recognition masking (ABRM) tasks. In both paradigms, the interstimulus interval (ISI) between tones in a pair was either short $(20 \mathrm{~ms}$ ) or long $(200 \mathrm{~ms})$. Temporal order discrimination was better for both groups of listeners at long than at short ISIs, but no group differences in performance were observed at either ISI. Performance on the ABRM task was also better at long than at short ISls and was influenced by variability in masker frequency and by the spectral proximity of target and masker. The only significant group difference was found in one condition of the ABRM task when the target-masker interval was $200 \mathrm{~ms}$, but this difference was not reliable when the measure was of optimal performance. Moderate correlations were observed between auditory thresholds and phonological skill for the sample as a whole and within the dyslexic and control groups. However, although a small subgroup of dyslexic listeners with poor phonology was characterized by elevated thresholds across the auditory tasks, evidence for an association between auditory and phonological processing skills was weakened by the finding of a subgroup of control listeners with poor auditory processing and normal phonological processing skills.

KEY WORDS: dyslexia, adults, phonological processing, rapid auditory processing, frequency discrimination

$\mathrm{n}$ recent years, there has been renewed interest in the possibility that a fundamental perceptual limitation in the auditory domain compromises phonological representations that are critical to oral and written language development (Tallal, Allard, Miller, \& Curtiss, 1997). This proposal followed from a series of experiments, conducted by Tallal and colleagues with children with specific language impairment (SLI), that made extensive use of an auditory repetition task. In this task, children were first taught to label differentially a pair of complex tones differing in fundamental frequency. Next, children were presented with sequences of the tones and had to indicate the order in which the tones had been presented. In the test phase, the interstimulus interval (ISI) for the tone pairs was varied from short to long durations.

Performance on this temporal order task was impaired for children in the SLI group when the ISI was relatively short (below $150 \mathrm{~ms}$; Tallal 
$\&$ Piercy, 1973a) and also when tones were short (75 $\mathrm{ms})$ rather than long $(250 \mathrm{~ms})$ in duration (Tallal \& Piercy, 1973b). Furthermore, the groups did not differ on a visual version of the task. The results were taken to indicate a deficit, specific to the auditory domain, in processing brief, rapidly presented auditory signals among children with SLI. ${ }^{1}$ Similar findings were reported for a group of 20 children with reading disability, who were also given a discrimination and a repetition version of the auditory task, using mixed presentation of pairs of stimuli at different ISIs (Tallal, 1980). It is important to note that only $40 \%$ (8 out of 20 ) of the poor readers showed a clear deficit on the repetition task.

Attempts to replicate Tallal's (1980) finding of rapid auditory processing deficits in samples of dyslexic children and adults have been equivocal (e.g., Heath, Hogben, \& Clark, 1999). In line with Tallal, Reed (1989) demonstrated group differences in performance on the repetition task for short (10 ms), but not for long (400 ms) ISIs (cf. Cacace, McFarland, Ouimet, Schrieber, \& Marro, 2000). Further studies have reported weaker levels of performance on the auditory repetition task for groups of dyslexic children, for both long and short ISIs (Marshall, Snowling, \& Bailey, 2001; Nittrouer, 1999; Reed, 1989; Waber et al., 2001).

A second experimental procedure used to investigate processing of rapidly presented auditory signals has involved auditory backward recognition masking (ABRM; McArthur \& Hogben, 2001). In this task individuals are required to discriminate and identify intensity or pitch differences between suprathreshold test signals that are followed by an auditory mask (Kallman \& Massaro, 1979; Massaro, 1972; Massaro \& Burke, 1991); recognition masking differs from energetic masking, which is manifest typically as elevated thresholds for signal detection. The effect of the masker on test recognition is greatest when the ISI between test and masker stimuli is relatively short $(250 \mathrm{~ms})$ and is reduced at longer ISIs because more time is available for perceptual processing of the signal before the arrival of the masker. This task can therefore be considered an index of rapid auditory perceptual processing on the assumption that more rapid processing of the signal will reduce the effects of the masker.

McArthur and Hogben (2001) used ABRM to examine the ability of reading-disabled children to make intensity discriminations between pairs of tones that were followed by an auditory mask. Overall, there were no differences in performance between poor readers and controls. However, a subgroup of the reading-disabled children showed elevated thresholds on the ABRM task;

\footnotetext{
${ }^{1}$ Tallal changed her position in later work (Tallal, 1980; Tallal et al., 1997), arguing for temporal order deficit; see Studdert-Kennedy and Mody (1995) for a discussion regarding the term temporal processing.
}

these poor readers tended to show concomitant oral language difficulties (cf. Heath et al., 1999). It should be noted, however, that at least some of the individuals with oral language difficulties in the study had ABRM thresholds within the normal range.

The question of what underlies deficits in performance on tests of rapid auditory processing remains unresolved. The finding that individuals with reading difficulties perform within the normal range on tasks typically taken to measure temporal resolution, such as the ability to detect a brief silent interval in ongoing noise (McAnally \& Stein, 1996; Schulte-Korne, Deimel, Bartling, \& Remschmidt, 1998), suggests that the impairment does not involve simply poor temporal resolution.

Several recent studies have reported that dyslexic listeners show deficits on tests tapping the discrimination of basic acoustic dimensions, including frequency discrimination, detection of frequency modulated tones, and binaural release from masking (Hari, Saaskilahti, Helenius, \& Uutela, 1999; McAnally \& Stein 1996). In a similar vein, Baldeweg, Richardson, and Gruzelier (1999) measured electroencephalic responses in groups of dyslexic and normal reading adults. They reported abnormal mismatch negativity in the dyslexia group in response to changes in tone frequency (but not to changes in tone duration) when performing a visual distractor task. In contrast, Hill, Bailey, Griffiths, and Snowling (1999) found no significant differences between adults with dyslexia and normal reading adults (matched for age and IQ) in mean frequency difference thresholds that were measured at relatively low $(1 \mathrm{kHz})$ or high $(6 \mathrm{kHz})$ frequencies. However, a subgroup of dyslexic listeners was noted to have high thresholds outside the range of performance for the control group at both frequencies. Conflicting findings have also been reported for samples of adults with dyslexia given tasks involving detection of frequency modulation (FM; Hill et al., 1999; Witton et al., 1998).

A plausible reason for at least some of the conflicting findings in studies investigating low-level auditory processes in dyslexia is that the dyslexic samples are drawn from different populations and may differ in severity. It is widely acknowledged that there are individual differences in dyslexic children's reading (Castles \& Coltheart, 1993) and related variations in the severity of their phonological processing deficits (Griffiths \& Snowling, 2002). Indeed, according to Tallal (1980), only those individuals demonstrating more severe phonological processing impairments would be expected to demonstrate deficits in rapid auditory processing.

In line with this view, correlations of moderate size have been reported for poor and normal readers between nonword reading (i.e., phonological decoding skill) and FM detection thresholds (Witton et al., 1998) and between 
nonword reading and performance on an auditory temporal order judgment task (Heath et al., 1999). McAnally, Castles, and Stuart (2000) concluded that frequency discrimination skill is associated specifically with nonword rather than word reading skill. Correlations between auditory performance and nonword reading have also been reported in unselected samples of normal readers, after controlling for the effects of age and IQ (Ahissar, Protopapas, Reid, \& Merzenich, 2000; Au \& Lovegrove, 2001; Talcott et al., 1999). However, Bishop, Carlyon, Deeks, and Bishop (1999) failed to find a significant correlation between nonword reading and auditory processing performance in a sample of children with SLI.

A more stringent test of the strength of the association between auditory processing and phonological processing skill is provided by its relation with measures of oral phonological processing rather than nonword reading. Marshall et al. (2001) reported a significant partial correlation after controlling for age and nonverbal ability between performance on the auditory repetition task and scores on tests of phoneme deletion, rhyme oddity, and nonword repetition for a group of dyslexic and normal readers. However, the relatively small samples of participants in these studies indicate a need for caution when interpreting significant correlations between auditory and phonological skills.

The present article was concerned principally with the hypothesis that people with dyslexia have a deficit that impairs their ability to process auditory stimuli presented in rapid succession (e.g., Tallal, 1980). A secondary objective was to examine whether our previous finding of unimpaired pure-tone frequency discrimination in adults with dyslexia (Hill et al., 1999) could be replicated using a larger sample and tones of much shorter duration. In line with Hill et al., our sample of adults with dyslexia consisted of high-functioning individuals selected from the student population. Although such samples of adults with dyslexia are not representative of the dyslexia population at large, our aim was to reduce the likelihood of introducing the unwanted confound of another comorbid disorder (e.g., SLI or attention deficit hyperactivity disorder [ADHD]; cf. McArthur, Hogben, Edwards, Heath, \& Mengler, 2001; Ramus et al., 2003).

In two experiments, we assessed the performance of dyslexic and normally reading adults on tasks involving "oddity" judgements about sequences of auditory stimuli presented at slow (200-ms ISI) and fast (20-ms ISI) rates of presentation. The aim was to extend the kinds of methods that have been used with children to adults. In each case, evidence was also sought for a relation between auditory processing and phonological processing skills. The first experiment used a temporal order discrimination (TOD) procedure in which listeners were presented with a sequence of four pairs of brief tones. There was a frequency difference $(\Delta F)$ between the two tones in each pair that was manipulated during the experiment. In three of the pairs within the sequence, the standard within-pair tone order was low-high, and in the target pair the order was high-low. The hypothesis that dyslexic listeners have an impairment in auditory processing of rapidly presented auditory events predicts that any differences between the dyslexic listeners and the normal-reading control listeners in the observed threshold should be greater in the short than in the long ISI condition.

The second experiment used an ABRM procedure to examine the degree to which dyslexic and control listeners could extract frequency information from the leading tone of a pair of brief tones. Listeners were required to detect the "odd-one-out" from a sequence of four test tones. Three of the four tones were at a standard frequency $(1 \mathrm{kHz})$ and the target tone was at a higher frequency (by $\Delta F \mathrm{~Hz}$ ). In a baseline condition there was no masking tone. In the other conditions in the experiment, each test tone was followed by a masking tone that was irrelevant to the task. There were two independent variables: (a) the duration of the interval between the test and masker tones (20 ms or $200 \mathrm{~ms}$ ) and (b) the frequency of the masking tone. The latter variable was fixed at the standard frequency, selected randomly from a range around the standard frequency, or selected randomly from a range an octave above the standard frequency.

On the basis of our previous results (Hill et al., 1999), we did not expect to find differences between dyslexic and control listeners on the frequency discrimination baseline condition, although, as noted above, some other studies have reported elevated frequency difference thresholds for dyslexic listeners. In the masker conditions, a masking tone would be expected to have an effect on the dependent variable provided that it (a) interferes with perceptual processing of a test tone and (b) arrives before the listener has extracted sufficient perceptual information for the task from the auditory representation of the test tone. Because these prerequisites should be met in masking conditions when test and masking tones are separated by the short ISI (Massaro, 1970; Sparks, 1976), it was predicted that recognition of the test tone frequency should be adversely affected in the short ISI condition relative to the long ISI condition. Furthermore, if dyslexic listeners are impaired in their ability to process rapidly presented auditory events then they should be more susceptible to the effects of the masker. This susceptibility should be greater at the short ISI and should manifest as greater threshold elevation (larger $\Delta F$ relative to 
the no-masker baseline condition) for dyslexic listeners than for control listeners. ${ }^{2}$

Different patterns of data may be expected for the different masker conditions. In the fixed-frequency masker condition (which has some similarities to the same-different discrimination test used by Tallal, 1980), masking effects may be at least partially offset by strategic use of within-pair test-masker comparisons to facilitate identification of the interval containing the target tone. When the frequency of the masker tone is varied randomly around the standard tone frequency-the $1-\mathrm{kHz}$ random-frequency masker condition-the potential benefits of strategic test-masker comparisons are eliminated. Instead, there is now a potential cost associated with the risk of confusing test and masking tones. The risk of this confusion is reduced when the masker frequency varies randomly around $2 \mathrm{kHz}$. These considerations predict magnitudes of masking effects in the following order: fixed-frequency masker $<2-\mathrm{kHz}$ random-frequency masker $<$ $1-\mathrm{kHz}$ random-frequency masker.

There are also grounds for expecting a difference in performance between dyslexic and control listeners across the two random-frequency masker conditions. Rosen and Manganari (2001) reported that people with dyslexia had higher detection thresholds than control listeners in a backward-masking task when the masker was a bandpass noise centered on the signal frequency. However, there was no significant difference in threshold when the masker energy was spectrally remote from the signal. Although the reason underlying the poorer performance of the people with dyslexia in the on-frequency masker

${ }^{2}$ Note that in using these masking-like effects as a performance metric, we made no assumptions about the mechanism by which masking tones have their effects. condition is unclear, this result provides some basis for predicting that the relative performance of dyslexic listeners will be poorer when the random frequency masker is varied around $1 \mathrm{kHz}$. The same prediction is suggested by Helenius, Uutela, and Hari's (1999) report that adults with dyslexia were more likely than control listeners to segregate a sequence of different-frequency tones into separate perceptual streams.

Thus, in all masker conditions for which the duration of the interval between test and masking tones is short, the hypothesis that people with dyslexia are impaired in processing rapidly occurring auditory events predicts poorer performance (manifest as elevated thresholds) for dyslexic listeners compared to control listeners.

\section{Method \\ Participants}

Twenty adults with dyslexia (8 men, 12 women), sampled from the student population at the University of York, participated in the study; all had a documented history of reading and spelling difficulties. Participants came from different academic departments and were studying a variety of subjects. The control group of 20 participants ( 8 men, 12 women) was also selected from a range of academic departments and subject disciplines and had no known history of difficulties with literacy acquisition. All participants had audiometric pure-tone thresholds not exceeding $20 \mathrm{~dB}$ HL in the range of 250 $8000 \mathrm{~Hz}$.

\section{Screening Variables}

Confirmation of participant status was based on data summarized in Table 1. The ages of the dyslexic

Table 1. Performance of dyslexic readers and controls on screening tests and additional tests of reading fluency and phonological skill

\begin{tabular}{|c|c|c|c|c|c|c|c|c|c|c|c|c|c|c|}
\hline \multirow[b]{2}{*}{ Group } & \multirow[b]{2}{*}{ Age } & \multicolumn{2}{|c|}{ IQ (WAIS-R) } & \multicolumn{2}{|c|}{$\begin{array}{l}\text { Literacy } \\
\text { (WRAT-III) }\end{array}$} & \multicolumn{2}{|c|}{$\begin{array}{l}\text { Reading } \\
\text { fluency } \\
\text { (TOWRE) }\end{array}$} & \multicolumn{2}{|c|}{$\begin{array}{l}\text { Nonsense } \\
\text { passage } \\
\text { reading }\end{array}$} & \multicolumn{2}{|c|}{ Spoonerisms } & \multicolumn{2}{|c|}{ RAN } & \multirow{2}{*}{$\begin{array}{c}\begin{array}{c}\text { Digit } \\
\text { span }\end{array} \\
\text { Scaled } \\
\text { score }\end{array}$} \\
\hline & & $\begin{array}{l}\text { Vocab } \\
\text { (Sc) }\end{array}$ & $\begin{array}{l}\text { Design } \\
\text { (Sc) }\end{array}$ & $\begin{array}{c}\text { Read } \\
\text { (SS) }\end{array}$ & $\begin{array}{l}\text { Spell } \\
\text { (SS) }\end{array}$ & Word & Nonword & $\begin{array}{l}\text { Time } \\
\text { (s) }\end{array}$ & $\begin{array}{l}\text { Total } \\
\text { errors }\end{array}$ & $\begin{array}{c}\text { Time } \\
\text { (s/item) }\end{array}$ & $\begin{array}{c}\text { Correct/ } \\
24\end{array}$ & $\begin{array}{l}\text { Digit } \\
\text { (ms) }\end{array}$ & $\begin{array}{l}\text { Object } \\
\text { (ms) }\end{array}$ & \\
\hline \multicolumn{15}{|c|}{ Dyslexic $(n=20)$} \\
\hline$M$ & 21 & 13 & 14 & 104 & 96 & 86 & 87 & 79 & 10 & 2.3 & 19 & 42 & 69 & 9 \\
\hline$S D$ & 2 & 2 & 3 & 8 & 9 & 12 & 8 & 38 & 4 & 1.0 & 6 & 10 & 10 & 2 \\
\hline \multicolumn{15}{|c|}{ Control $(n=20)$} \\
\hline$M$ & 22 & 15 & 14 & 111 & 113 & 108 & 107 & 45 & 3 & 1.1 & 23 & 28 & 56 & 12 \\
\hline$S D$ & 5 & 2 & 2 & 5 & 7 & 9 & 9 & 10 & 3 & 0.5 & 1 & 7 & 10 & 3 \\
\hline
\end{tabular}

Note. WAIS-R = Wechsler Adult Intelligence Scale-Revised; WRAT-III = Wide Range Achievement Test-Third Edition; TOWRE = Test of Word Reading Efficiency; RAN = rapid automatized naming; Sc = scaled scores; SS = standard scores. 
and the control readers ranged from 18.5 to 28.8 years and from 18.5 to 36.9 years, respectively. Matching for IQ was on the basis of the Block Design and Vocabulary subtests of the Wechsler Adult Intelligence ScaleRevised (WAIS-R; Wechsler, 1994). The two groups did not differ in age or IQ: $F(1,38)=2.9, n s$, for Vocabulary; $F<1$ for Block Design.

Attainments in reading and spelling accuracy were obtained from all participants by administration of the Wide Range Achievement Test-Third Edition (WRATIII; Jastak \& Wilkinson, 1993). The reading attainments of the dyslexia group were age-appropriate; however, given their relatively high level of intelligence, their reading level was below expectation (see Table 1). Reading and spelling raw scores for the dyslexia group were poorer statistically than those of the control group: $F$ (1, $38)=14.0, p<.001$, for reading; $F(1,38)=40.7, p<.001$, for spelling.

\section{Literacy and Phonological Processing Measures}

Additional measures of reading and phonological skill were administered to all participants to confirm group status (Hatcher, Snowling, \& Griffiths, 2002).

\section{Test of Word Reading Efficiency (TOWRE)}

The TOWRE (Torgeson et al., 1999) was used to measure reading rate and accuracy. Participants were shown a list of either words or nonwords and instructed to read aloud as many items as possible within $45 \mathrm{~s}$.

\section{Nonsense Passage Reading}

Nonsense passage reading was chosen to assess decoding skill. Each participant read two passages taken from Finucci et al. (1976, cited in Gross-Glenn, Jallad, Novoa, Helgren-Lempesis, \& Lubs, 1990). The first passage contained 17 nonwords embedded in a context of 52 words (e.g., "Once upon a time a tawndy rapsig named Gub found a tix of pertollic asquees."). The second passage contained 13 nonwords embedded in a context of 44 words. Reading time and accuracy were recorded.

\section{Spoonerisms}

This task assessed participants' abilities to segment and manipulate phonemes by asking them to exchange the beginning sounds of two words. The words were the first and last names of famous individuals. Following training and practice items, 12 test items were administered; accuracy and response time measures were recorded.

\section{Rapid Naming}

Rapid automatized naming (RAN) was assessed using two tasks from the Phonological Assessment Battery (Frederickson, Frith, \& Reason, 1997): Digit Naming and Object Naming. Participants were required to name, respectively, 50 digits and 50 pictures of five objects presented on two cards in random order. Performance was recorded as the time taken to name the items, to the nearest second.

\section{Verbal Short-Term Memory}

The Digit-Span subtest from the WAIS-R (Wechsler, 1994) was used to assess short-term memory, administered according to the test manual.

\section{Auditory Tasks Stimuli and Equipment}

The stimuli were pure tones having a total duration of $75 \mathrm{~ms}$, including 5 -ms linear onset and offset ramps. All tones were presented at a level of $65 \mathrm{~dB}$ SPL. Stimulus waveforms were computed in software using 24-bit amplitude quantization and a sampling frequency of $44.1 \mathrm{kHz}$. The waveforms were converted into voltages by means of a LynxOne 24-bit audio card (Lynx Studio Technology, Inc., Costa Mesa, CA). The resulting analog signals were routed to a pair of Sennheiser HD580 headphones situated inside an IAC 1201-A double-walled sound-attenuating booth. A flat panel LCD monitor inside the booth provided listeners with information about the status of the experiment. Responses were made via a standard computer keyboard. The experiments were controlled using custom software running on an IBMcompatible PC. The level and duration of the tones was the same for the TOD and ABRM tasks.

\section{Temporal Order Discrimination (TOD)}

Each trial began with the presentation of a visual alerting stimulus for $400 \mathrm{~ms}$. Listeners then were presented with four pairs of tones $400 \mathrm{~ms}$ after the offset of the alerting stimulus. The time interval between the onsets of the leading tone in successive pairs was fixed at $775 \mathrm{~ms}$, while the silent interval between members of a pair was either 20 or $200 \mathrm{~ms}$. The two conditions were tested in separate blocks, with order of testing randomized across participants. Three of the tone pairs were presented with a standard frequency tone leading a tone of higher frequency, with the remaining target pair having the reverse order. The target pair was always presented in the second or third interval with equal a priori probability, and the task of the listener was to identify the interval containing the target. Listeners were given an unlimited time in which to make a response, after 
which they were provided with visual feedback for 400 $\mathrm{ms}$. An interval of $1 \mathrm{~s}$ separated the offset of the feedback signal and the start of the next trial.

The standard frequency tone in each pair was fixed at $1 \mathrm{kHz}$. The frequency of the other tone was varied adaptively over a 60 -trial run according to a two-down, one-up rule that converged on the frequency difference corresponding to $71 \%$ correct discrimination (Levitt, 1971). At the beginning of a run, the frequency difference between the two tones in each pair (relative to the standard frequency of $1 \mathrm{kHz}$ ) was $8 \%$. This difference was adjusted using an initial multiplier of 0.5 (i.e., two correct responses resulted in a halving of the frequency difference, whereas an incorrect response resulted in the difference being doubled). The multiplier was increased to 0.7 following the first four reversals to improve the resolution of the procedure near threshold. Errors on the first trial were discarded and did not count as a reversal. The threshold for a single run was defined as the geometric mean of the frequency difference at each reversal after the first four reversals (three if the total number of reversals was odd). Each listener completed four runs per condition, and the threshold for each condition was taken as the geometric mean of the four runs.

\section{Auditory Backward Recognition Masking (ABRM)}

Frequency difference limens (DLFs) were estimated for each listener for test tones presented alone without a masking tone (the baseline condition). The task was presented using a four-interval, two-alternative, forcedchoice (oddity) procedure, with the target signal presented with equal a priori probability in either the second or third interval. The frequency of the tones in three of the four intervals was fixed at the $1-\mathrm{kHz}$ standard, and the frequency of the target tone was adjusted adaptively using the same parameters as for the TOD task.

In the remaining conditions, DLFs were estimated for backward-masked tones. On each trial, the test tone was the leading tone in each pair, followed by a masker tone that listeners were instructed to ignore. For three of the four tone pairs, the frequency of the leading tone was fixed at $1 \mathrm{kHz}$. For the remaining pair, the leading (target) tone was higher in frequency. The task of the listener was to identify the interval in which the leading tone had a frequency greater than $1 \mathrm{kHz}$, which was always the second or third interval with equal a priori probability. On each presentation, the frequency of the masker tone in each pair was (a) fixed at $1 \mathrm{kHz}$, (b) roved in frequency over the range of $1.8-2.2 \mathrm{kHz}$, or (c) roved in frequency over the range of $0.9-1.1 \mathrm{kHz}$. In the latter two conditions, roved in frequency implies that a new masker frequency was selected randomly from the specified range for each masker presentation. The three masker conditions were tested in separate blocks of four 60 -trial runs. Listeners were first tested using an ISI of $200 \mathrm{~ms}$ (between members of a pair) before being tested on a condition in which the ISI was $20 \mathrm{~ms}$. The adaptive procedure used to estimate threshold was the same as that described above. Again, each listener completed four runs per condition and the threshold for each condition was taken as the geometric mean of the four runs.

\section{Procedure}

In the first session, participants received the cognitive test battery and were screened for hearing loss using standard pure-tone audiometry. Participants completed the TOD task and baseline ABRM condition in the same 2 -hr session, with a 15 -min break between the two tasks. The remaining ABRM conditions were completed in two separate 2-hr sessions: in the first of these sessions the ISI was $200 \mathrm{~ms}$ (three masker conditions, in separate blocks), and in the final session the ISI was $20 \mathrm{~ms}$ (three masker conditions, in separate blocks). The three-masker conditions were presented in a fixed order for each ISI: fixed masker, random-frequency masker centered around $2 \mathrm{kHz}$, random-frequency masker centered around $1 \mathrm{kHz}$. For an individual participant, no more than one session was completed per day, all four sessions being completed within a maximum period of 1 month.

\section{Results \\ Reading and Phonological Skills}

The performance of the dyslexic and control groups on the measures of reading and phonological processing skills is summarized in Table 1. Multivariate analyses of variance revealed that the dyslexia group performed significantly less well than the control group on the reading measures, $\Delta(6,28)=9.8, p<.001$, and on the phonological processing tasks, $\Delta(8,35)=4.32, p$ $<.001$. Univariate tests confirmed significant group differences on each of the reading and phonological measures $(p<.01)$.

\section{TOD}

Mean TOD thresholds for each group of listeners and individual thresholds for each listener are shown in Figure 1. Reported thresholds are expressed as a percentage of the standard frequency. Both groups had higher thresholds in the 20-ms ISI condition than in the 200-ms ISI condition (standard deviations were similar for each group). TOD thresholds for the dyslexia group were slightly higher than those for the control group in both ISI conditions (20-ms ISI: dyslexia group $=0.86 \%$, control group $=0.76 \% ; 200-\mathrm{ms}$ ISI: dyslexia group $=$ $0.73 \%$, control group $=0.64 \%$ ). 
Figure 1. Dyslexic and normal readers' performance on the TOD task for interstimulus intervals (ISIs) of $200 \mathrm{~ms}$ (circles) and 20 ms (diamonds). Thresholds are expressed as a percentage of standard frequency. Individual listener thresholds for each group are shown by the smaller symbols; the enlarged points for each stimulus condition represent the geometric mean threshold for each group.

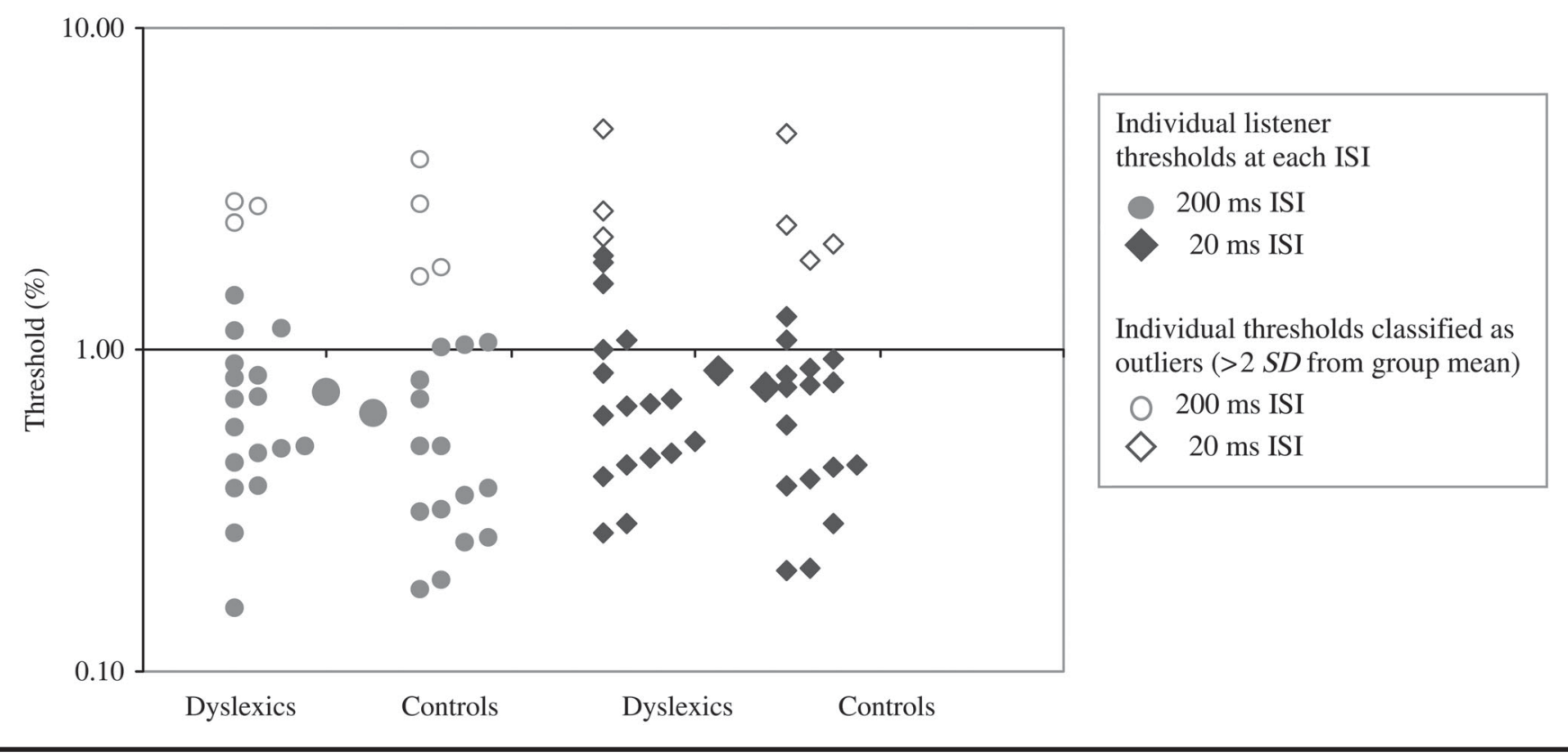

An analysis of variance (ANOVA) was performed using a logarithmic transformation of these data, with group (dyslexia, control) as a between-subjects factor and ISI $(200 \mathrm{~ms}, 20 \mathrm{~ms})$ as a within-subjects factor. There was no main effect of group $(F<1)$, but there was a significant main effect of ISI, $F(1,38)=4.9, p<.05$. There was no significant Group $\times$ ISI interaction $(F<1)$.

Inspection of the individual thresholds in each condition of this experiment revealed some noteworthy patterns of individual variation within the sample. A subgroup of 5 individuals with dyslexia and 4 controls showed elevated thresholds on this task at both ISIs relative to the group mean. Thresholds ranged from $1.17 \%$ to $4.86 \%$ for the adults in the dyslexia subgroup and from $1.27 \%$ to $4.68 \%$ for adults in the control subgroup. Otherwise, there was almost complete overlap of the TOD thresholds between the group with dyslexia and the control group.

\section{ABRM}

The results of the dyslexic and normal reader groups on the ABRM task are shown in Figure 2. Analyses were performed using a logarithmic transformation of these data.

\section{Baseline Condition}

The baseline condition yielded an estimate of the DLF for $1-\mathrm{kHz} 75-\mathrm{ms}$ pure tones. Mean DLFs were similar in magnitude for the two groups of listeners (dyslexia group $=0.42 \%$; control group $=0.52 \%$, but were numerically larger for dyslexic listeners than for the controls, with standard deviations of similar magnitude for both groups. A one-way ANOVA showed that the DLFs for the two groups did not differ reliably $(F<1)$. No outliers were identified in the dyslexia group (using a criterion of $\pm 1 S D$ from the mean), but 1 individual was identified as an outlier in the control group.

\section{Masker Conditions}

To examine the effects of masker type and targetmasker ISI on threshold estimates, we performed a $2 \times$ $2 \times 3$ mixed-design ANOVA, with group (dyslexia, control) as a between-subjects factor and masker type (fixed, $2 \mathrm{kHz}, 1 \mathrm{kHz}$ ) and ISI ( $200 \mathrm{~ms}, 20 \mathrm{~ms}$ ) as withinsubjects factors. The difference between the two groups did not reach statistical significance, $F(1,38)=3.6, p=$ .06 , but there were significant main effects of both masker type, $F(2,76)=171.55, p<.001$, and ISI, $F(1$, $38)=68.1, p<.001$. Both groups of listeners had higher thresholds in the short ISI condition than in the longer ISI condition. Tests of simple contrasts were used to show that mean thresholds differed significantly for each of the following contrasts: fixed $<$ random $2-\mathrm{kHz}$ masker $<$ random $1-\mathrm{kHz}$ masker.

The Group $\times$ ISI interaction was not significant $(F$ $<1$ ), but the Group $\times$ Masker interaction was significant, $F(2,76)=3.7, p<.05$. Tests of simple main effects indicated that dyslexic listeners had higher thresholds 
Figure 2. Dyslexic and normal readers' performance on the ABRM task (Experiment 2) for ISls of $200 \mathrm{~ms}$ (left panel) and $20 \mathrm{~ms}$ (right panel). Thresholds are expressed as a percentage of the standard frequency. The error bars denote standard deviations of the mean. DLF $=$ frequency difference limen.

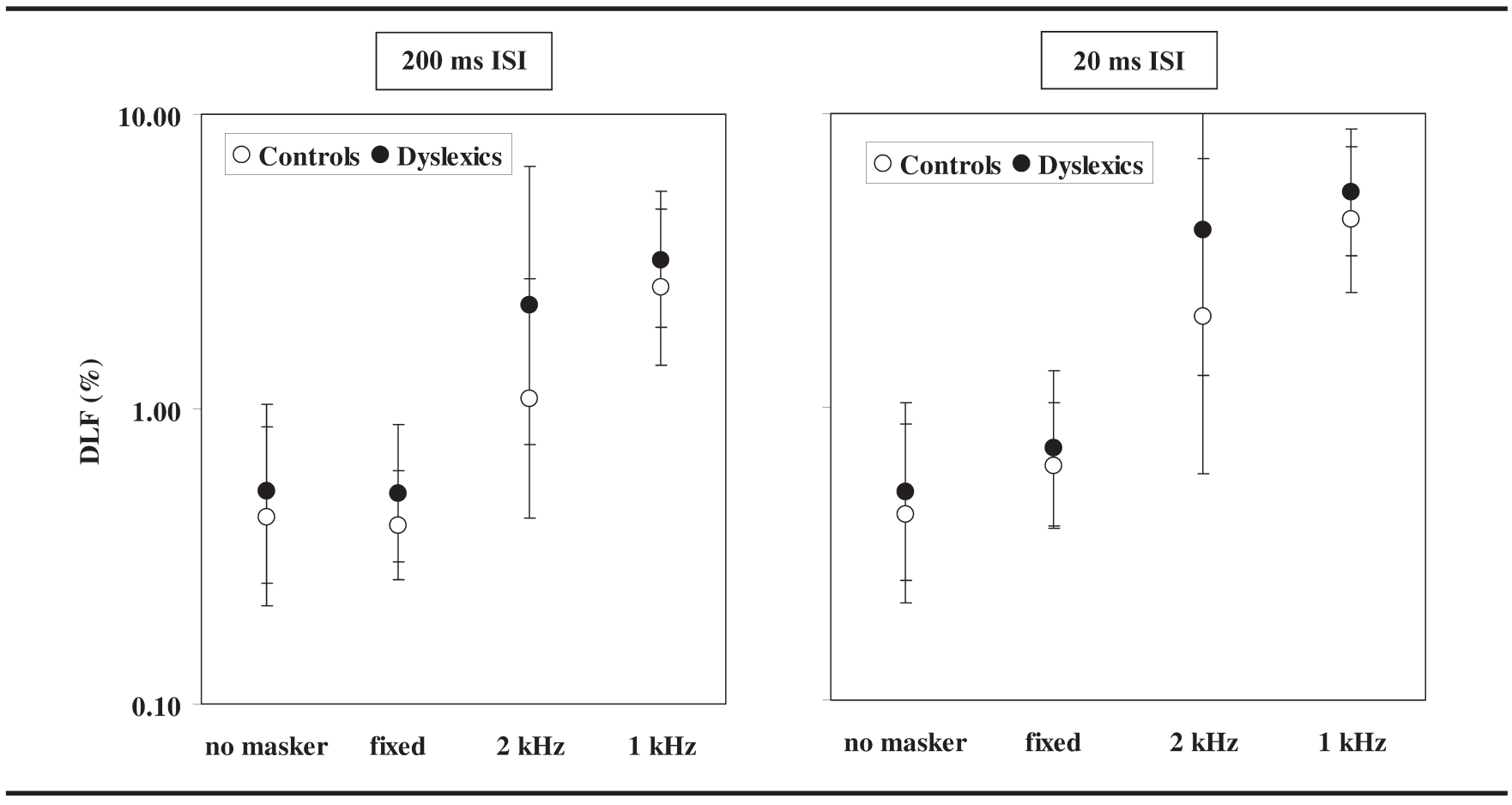

than controls in the $2-\mathrm{kHz}$ random-masker condition (group with dyslexia $=3.00 \%$; control group $=1.60 \%$ ), but the groups did not differ in the fixed-masker or in the $1-\mathrm{kHz}$ random-masker conditions. For dyslexic listeners, thresholds in the fixed-masker condition were reliably lower than those in the two random-masker conditions, which did not differ (fixed $=0.61 \%, 2 \mathrm{kHz}=$ $3.00 \%, 1 \mathrm{kHz}=4.17 \%$ ). For control listeners, thresholds also were reliably lower in the fixed-masker condition than in the random-masker conditions (fixed = $0.50 \%, 2 \mathrm{kHz}=1.49 \%, 1 \mathrm{kHz}=3.36 \%$ ), and, unlike dyslexic listeners, control listeners' thresholds in the $2-\mathrm{kHz}$ random-masker conditions were reliably lower than those in the $1-\mathrm{kHz}$ random-masker condition $(p<.001)$.

Neither the Masker $\times$ ISI interaction, $F(2,76)=2.0$, $n s$, nor the Group $\times$ ISI $\times$ Masker interaction $(F<1)$ was significant. Thus, the effect of the different masker types on performance was similar at both short and long ISIs. We note, however, that thresholds were slightly elevated for both groups in the fixed-masker condition relative to the baseline condition for short, $F(1,38)=2.6, p<$ .001 , but not long ISIs $(F<1)$.

When the distribution of scores was examined for outliers, the same subgroups of individuals in both the dyslexic and the control group who were identified for the TOD task were also outliers in some (but not all) of the masker conditions. A small number of dyslexic (6 out of 20) and control listeners (5 out of 20) who had not been identified previously as outliers also had elevated thresholds relative to the rest of the sample in some (but not all) of the masker conditions.

\section{Optimal Thresholds}

For all of the above analyses, thresholds for individual participants had been calculated by averaging across the thresholds obtained for the four runs in each condition. Inspection of these thresholds for participants in both groups revealed considerable within-condition variation, raising the possibility that threshold estimates based on an average of the thresholds for individual runs might have obscured subtle differences in performance between the two groups of listeners. To assess this possibility, we reanalyzed these data taking as the measure of performance the lowest threshold estimate for each listener from the four runs in each condition. Mean performance based on these best thresholds is summarized for both groups of listeners in Table 2 .

In an ANOVA based on best thresholds, the main effect of group was not significant, $F(1,38)=3.75, p=$ .06 , although as before there was a tendency for the dyslexia group to have higher thresholds than normal readers (group geometric means [collapsed across conditions]: dyslexia $=0.77 \%$, control $=0.52 \%$ ). Significant main effects were found for both ISI, $F(1,38)=57.5, p<$ .001 , and masker type, $F(1.54,58.3)=117.71, p<.001$. There was a significant interaction between ISI and 
Table 2. Performance of dyslexic and control listeners in the ABRM task showing lowest $\%$ thresholds.

\begin{tabular}{|c|c|c|c|c|c|c|c|c|c|c|c|c|c|c|}
\hline \multirow[b]{3}{*}{ Group } & \multirow{2}{*}{\multicolumn{2}{|c|}{$\begin{array}{c}\text { No masker } \\
\text { (DLF 75) }\end{array}$}} & \multicolumn{4}{|c|}{ Fixed masker } & \multicolumn{4}{|c|}{ Random masker (2 kHz) } & \multicolumn{4}{|c|}{ Random masker (1 kHz) } \\
\hline & & & \multicolumn{2}{|c|}{200 ISI } & \multicolumn{2}{|c|}{20 ISI } & \multicolumn{2}{|c|}{200 ISI } & \multicolumn{2}{|c|}{20 ISI } & \multicolumn{2}{|c|}{200 ISI } & \multicolumn{2}{|c|}{20 ISI } \\
\hline & D & C & D & $C$ & D & C & D & C & D & $C$ & D & C & D & C \\
\hline \multicolumn{15}{|c|}{ Geometric } \\
\hline$M$ & 0.4 & 0.3 & 0.4 & 0.3 & 0.5 & 0.4 & 1.1 & 0.6 & 2.3 & 1.0 & 2.0 & 1.7 & 3.6 & 2.9 \\
\hline$S D^{*}$ & 0.3 & 0.4 & 0.2 & 0.2 & 0.3 & 0.3 & 0.5 & 0.4 & 0.5 & 0.6 & 0.3 & 0.3 & 0.2 & 0.3 \\
\hline
\end{tabular}

Note. $A B R M=$ auditory backward recognition masking; $D L F=$ frequency difference limen; $I S I=$ interstimulus interval; $D=d y$ slexia group; $C=$ control group. Standard deviations were calculated using log $X$ data.

masker type, $F(1.9,74.5)=3.6, p<.05$, but, as before, none of the other interactions by group was significant.

\section{Relationships Between Auditory, Phonological, and Reading Skills}

To investigate the relation between auditory mean thresholds and measures of phonological and literacy performance, we computed correlations between the reading and phonological processing variables and between these and mean thresholds from the two auditory processing tasks (TOD and ABRM) for the pooled data from dyslexic and normal readers (see the data below the diagonal in Table 3). Neither age nor Block Design correlated with any of the other measures; however, there were moderate correlations between vocabulary and reading and spelling accuracy $(r=.5-.7)$. The data above the diagonal in Table 3 show the partial correlations between the literacy and phonology variables after controlling for vocabulary levels in the sample. Because the pattern of results is essentially the same below and above the diagonal, all reported values are partial correlation coefficients controlled for vocabulary.

To reduce the data set before exploring the correlations between the phonological, literacy, and auditory processing measures, we derived two composite variables. These variables were justified by the intercorrelations among measures. ${ }^{3}$ The literacy variable consisted of performance on spelling accuracy (WRAT-III), word and nonword reading fluency (TOWRE word and nonword tasks), and both error and time measures from the nonsense passage reading task. Reading accuracy (WRAT-III) was not included in the composite because it correlated weakly (often not all) with phonological processing skill in this sample. The phonological variable consisted of performance on the spoonerisms task (average word response time), and digit and object naming speed (RAN tasks). Digit Span was not included in the composite score because it correlated weakly (or not at all) with the literacy measures.

${ }^{3} Z$ scores for each test relative to the sample as a whole were used to form composite scores that were the average of these $z$ scores.

Table 3. Intercorrelations between age, IQ, phonological skill, and literacy skill (below the diagonal) and partial correlation coefficients controlling for vocabulary (above the diagonal) for the full sample $(n=40)$.

\begin{tabular}{|c|c|c|c|c|c|c|c|c|c|c|c|c|c|c|}
\hline & & 1 & 2 & 3 & 4 & 5 & 6 & 7 & 8 & 9 & 10 & 11 & 12 & 13 \\
\hline 1 & Age & - & & .1 & .2 & .1 & -.0 & -.0 & -.1 & -.1 & .1 & .1 & .1 & .01 \\
\hline 2 & WAIS-R Vocabulary (raw) & .2 & - & & & & & & & & & & & \\
\hline 3 & WAIS-R Block design (raw) & .2 & .2 & - & .1 & .1 & .1 & -.1 & .0 & -.1 & -.1 & -.1 & -.3 & .1 \\
\hline 4 & WRAT Reading & .3 & $.5^{* *}$ & .2 & - & $.6^{* * *}$ & .2 & $.6^{* * *}$ & $-.6^{* * *}$ & $-.4^{*}$ & $-.3^{*}$ & $-.3^{*}$ & $-.5^{* *}$ & .2 \\
\hline 5 & WRAT Spelling & .2 & $.5^{* *}$ & .2 & $.7^{* * *}$ & - & $.5^{* *}$ & $.8^{* * *}$ & $-.8^{* * *}$ & $-.6^{* * *}$ & $-.6^{* * *}$ & $-.4^{*}$ & $-.7^{* * *}$ & $.4^{*}$ \\
\hline 6 & TOWRE words & .0 & .1 & .1 & .3 & $.5^{* *}$ & - & $.6^{* * *}$ & $.5^{* *}$ & -.3 & $-.5^{* *}$ & $-.6^{* * *}$ & $-.4^{*}$ & .2 \\
\hline 7 & TOWRE nonwords & .1 & .3 & -.0 & $.6^{* * *}$ & $.8^{* * *}$ & $.6^{* * *}$ & - & $-.8^{* * *}$ & $-.8^{* * *}$ & $-.6^{* * *}$ & $-.5^{* *}$ & $-.7^{* * *}$ & $.4^{*}$ \\
\hline 8 & Nonsense passage (errors) & -.2 & -.1 & .0 & $-.6^{* * *}$ & $-.8^{* * *}$ & $-.5^{* *}$ & $-.8^{* * *}$ & - & $.6^{* * *}$ & $.5^{* *}$ & $.6^{* * *}$ & $.7^{* * *}$ & -.3 \\
\hline 9 & Nonsense passage (time) & -.2 & -.2 & -.1 & $-.5^{* * *}$ & $-.7^{* * *}$ & $-.4^{*}$ & $-.8^{* * *}$ & $.6^{* * *}$ & - & $.5^{* *}$ & $.4^{*}$ & $.7^{* * *}$ & -.3 \\
\hline 10 & RAN digits & .1 & -.2 & -.1 & $-.4^{*}$ & $-.7^{* * *}$ & $-.5^{* *}$ & $-.7^{* * *}$ & $.5^{* *}$ & $.6^{* *}$ & - & $.6^{* * *}$ & $.7^{* * *}$ & $-.4^{*}$ \\
\hline 11 & RAN objects & .1 & -.1 & -.1 & $-.4^{*}$ & $-.4^{*}$ & -.6 & $-.5^{* * *}$ & $.6^{* * *}$ & $.4^{*}$ & $.6^{* * *}$ & - & $.5^{* *}$ & $-.6^{* * *}$ \\
\hline 12 & Spoonerisms (time) & .0 & -.1 & -.3 & $-.5^{* *}$ & $-.6 * * *$ & $-.4^{*}$ & $-.7^{* * *}$ & $.7^{* * *}$ & $.7^{* * *}$ & $.7^{* * *}$ & $.5^{* *}$ & - & $-.5^{* *}$ \\
\hline 13 & Digit span (raw) & .1 & $.3^{*}$ & .1 & $.3^{*}$ & $.5^{* * *}$ & .2 & $.4^{* *}$ & $-.4^{*}$ & $-.4^{*}$ & $-.4^{* *}$ & $-.6^{* * *}$ & $-.5^{* *}$ & - \\
\hline
\end{tabular}

${ }^{*} p<.05 . \quad{ }^{* *} p<.01 . \quad{ }^{* * *} p<.001$. 
Table 4. Partial correlations between literacy, phonology, and auditory measures, controlling for vocabulary for the dyslexic (below the diagonal) and the control group (above the diagonal).

\begin{tabular}{llcccccccc}
\hline & & $\mathbf{1}$ & $\mathbf{2}$ & $\mathbf{3}$ & $\mathbf{4}$ & $\mathbf{5}$ & $\mathbf{6}$ & $\mathbf{7}$ & $\mathbf{8}$ \\
\hline 1 & Literacy & - & $.7^{* *}$ & -.4 & -.5 & -.1 & -.4 & -.4 & $-.5^{*}$ \\
2 & Phonology & $.5^{*}$ & - & $-.5^{*}$ & $-.5^{*}$ & -.2 & -.3 & -.3 & $-.6^{*}$ \\
3 & TOD 20 & -.3 & -.5. & - & $.9^{* * *}$ & $.8^{* * *}$ & $.8^{* * *}$ & $.8^{* * *}$ & $.9^{* * *}$ \\
4 & TOD 200 & -.2 & -.4 & $.9^{* * *}$ & - & $.8^{* * *}$ & $.8^{* * *}$ & $.8^{* * *}$ & $.9^{* * *}$ \\
5 & DLF 75 & -.4 & -.5. & $.7^{* * *}$ & $.8^{* * *}$ & - & $.9^{* * *}$ & $.8^{* * *}$ & $.8^{* * *}$ \\
6 & ABRM fixedc & -.4 & -.3 & $.6^{* *}$ & $.8^{* * *}$ & $.8^{* * *}$ & - & $.9^{* * *}$ & $.7^{* * *}$ \\
7 & ABRM random 2c & -.1 & -.2 & $.7^{* *}$ & $.8^{* * *}$ & $.7^{* *}$ & $.7^{* * *}$ & - & $.8^{* * *}$ \\
8 & ABRM random 1c & -.3 & -.3 & $.5^{*}$ & $.7^{* *}$ & $.5^{*}$ & $.6^{* * *}$ & $.7^{* *}$ & - \\
\hline
\end{tabular}

Note. $T O D=$ temporal order discrimination.

aliteracy composite. 'b Phonology composite. 'Thresholds collapsed across ISI.

${ }^{*} p<.05 . \quad{ }^{* *} p<.01 . \quad{ }^{* * *} p<.001$.

Table 4 summarizes the partial correlation coefficients (controlling for vocabulary) between the literacy, phonology, and auditory measures for the dyslexia group (below the diagonal) and for the control group (above the diagonal). These results must be interpreted with caution due to the small sample size for each group ( $n=$ 20 ). For dyslexic readers, weak correlations were observed between the phonology variable and thresholds obtained in the TOD task, but the relation was only statistically significant for the short ISI. The phonology variable also correlated moderately with thresholds from the ABRM baseline condition $(r=-.5, p<.05)$. For the control group, moderate correlations were observed between the phonology variable and both conditions of the TOD task (20-ms ISI: $r=-.5, p<.05 ; 200$-ms ISI: $r=-.5$, $p<.05$ ), but phonology did not correlate with performance in the baseline ABRM condition, $r(20)=-.18$. The phonology variable correlated with thresholds from the random 1-kHz masker (collapsed across ISI; $r=-.6, p<.05$ ) in the control listener group only, but with and in none of the other masker conditions. Finally, moderate correlations between phonology and literacy were observed for both groups, with the coefficient for the dyslexia group $(r=.5)$ being smaller, but not significantly so, than that for the control group $(r=.7)\left(z_{\text {obt }}=-0.6, n s\right)$.

The relations between phonology composite scores ( $z$ scores) and estimated log thresholds for TOD and ABRM tasks (the latter collapsed across ISI) are shown in Figure 3. These scatterplots reveal that a subgroup of dyslexic individuals falls in the lower right quadrant of these plots, with weak phonological and auditory processing ability relative to the rest of the individuals in the sample. If we adopt a criterion of thresholds more than $1 S D$ from the mean, then 3 dyslexic individuals were consistently classified as outliers on both TOD and ABRM tasks. However, there was also in each case a subgroup of control participants who had relatively high thresholds but good phonological processing ability relative to the sample as a whole (see the upper right quadrant of the plots). Using the same outlier criterion, 4 control participants were classified as outliers across both tasks.

\section{$\overline{\text { Discussion }}$}

In this study we set out to evaluate the proposal that phonological processing impairments in dyslexia can be traced to more basic auditory processing deficits. The adults with dyslexia who participated in the study showed poor levels of literacy and phonological processing when compared to a group of age- and IQ-matched students without dyslexia. Both groups of adult listeners completed two tasks examining the rapid auditory processing of sequential stimuli. The first, the TOD task, addressed dyslexic listeners' ability to discriminate the temporal order of tones differing in pitch at varying rates of presentation. The second, the ABRM task, addressed the dyslexic listeners' abilities to discriminate tone frequency under conditions when individual tones were followed by masking tones at short target-masker intervals.

In the TOD task, both groups of listeners found it more difficult to make temporal order discriminations of pitch in the 20-ms ISI condition than in the $200-\mathrm{ms}$ ISI condition. Although mean thresholds for dyslexic listeners were numerically higher than those for control listeners in both conditions, thresholds for the two groups did not differ significantly. Furthermore, although a subgroup of listeners with elevated thresholds in both conditions was identified, both dyslexic and control listeners were members of this subgroup. The position taken by Tallal and colleagues (1997) predicts that individuals with TOD deficits should have the most impaired levels of phonological processing in the sample. Our data are not consistent with this position; specifically, 
Figure 3. These scatter plots summarize the relations between phonological skills and auditory processing measures (collapsed across ISI). Upper left panel: phonology composite score ( $z$ scores) plotted against log thresholds from the TOD task; upper right panel: phonology composite score plotted against DLFs (log thresholds) from the ABRM task; lower left panel: phonology composite score plotted against log thresholds from random $2-\mathrm{kHz}$ masker condition; lower right panel: phonology composite score plotted against log thresholds from random $1-\mathrm{kHz}$ masker conditions. Linear fits to the whole sample are shown in each case.

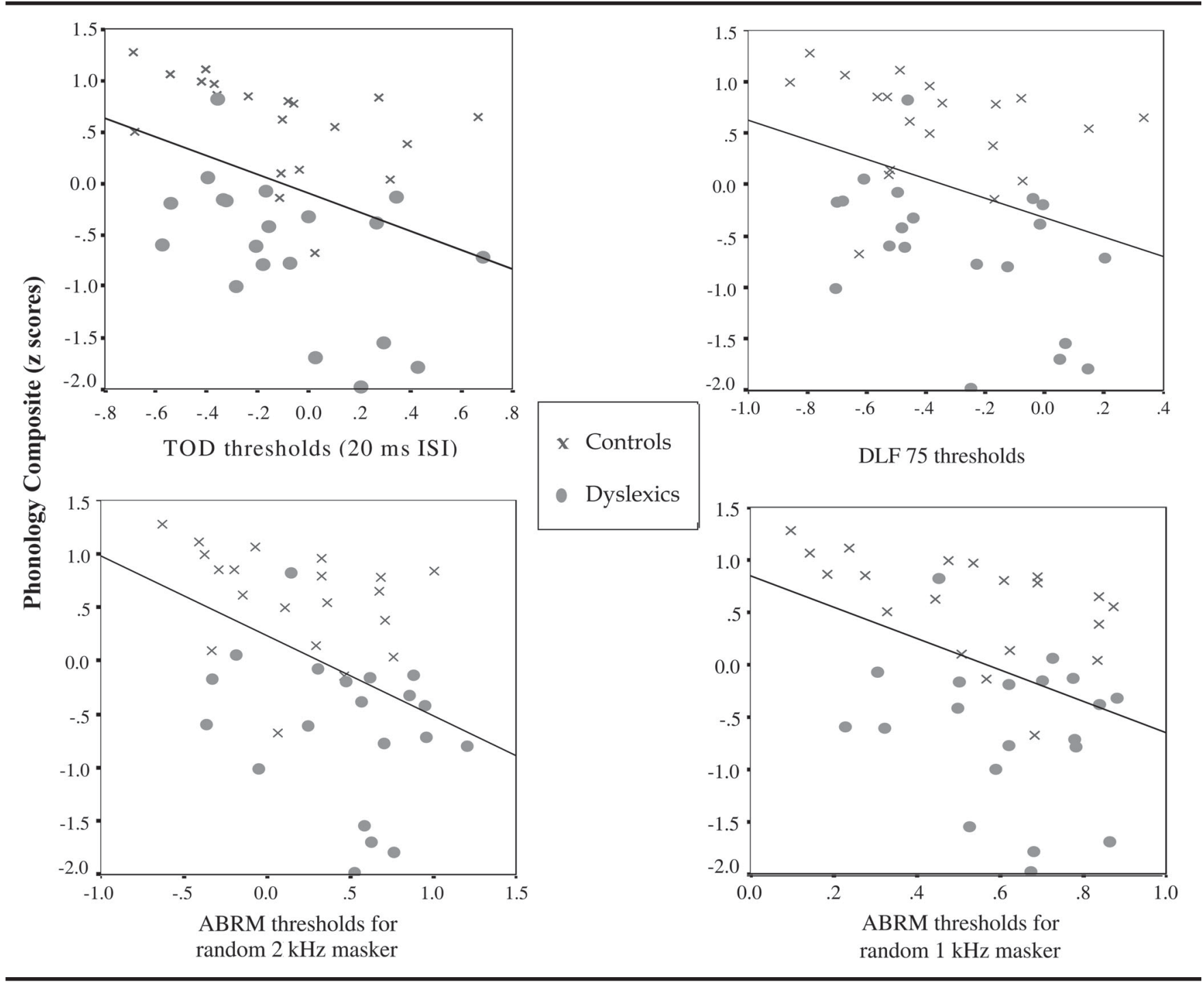

a subgroup of control listeners who showed impaired levels of performance on the TOD task demonstrated normal levels of phonological processing skill, and there were other adults with dyslexia in the sample with severe phonological impairments whose thresholds on the TOD task fell within the normal range.

An alternative hypothesis, based on the findings of Heath et al. (1999) and McArthur and Hogben (2001), predicts that the subgroup of dyslexic individuals with elevated thresholds would demonstrate concomitant oral language difficulties. For practical reasons, it was not possible to administer similar tests to those used with children in this study. However, the individuals classified as outliers all obtained either average or above average vocabulary scores, ruling this out as a plausible explanation.

Taken together, our findings with adult listeners contrast with those of studies reporting significant reader-group differences on TOD tasks in children at both short and long ISIs (Heath et al., 1999; Marshall et al., 2001; Nittrouer, 1999; Waber et al., 2001). Moreover, the results provide no support for the hypothesis that dyslexic readers have a specific deficit in the TOD of rapidly presented tones at short ISIs (Ahissar et al., 2000; Cacace et al., 2000; Heath et al., 1999; Reed, 1989; Tallal, 1980; Tallal \& Stark, 1982).

The second experimental task used the ABRM paradigm to assess rapid auditory processing. Specifically, 
we used the ABRM paradigm to explore the effects of manipulating target-masker intervals on frequency discrimination. As in the TOD task, thresholds for all listeners were significantly influenced by the duration of the ISI, with a significant elevation in overall thresholds in the $20-\mathrm{ms}$ ISI condition relative to the $200-\mathrm{ms}$ ISI condition. However, the nonsignificant interaction between group and ISI again failed to support the claim that dyslexic individuals have a specific rapid auditory processing deficit. The similarity in magnitude of estimates of DLFs for dyslexic and control listeners in the baseline condition is consistent with our previous results showing no group differences in frequency discrimination performance for longer duration tones and a different sample of dyslexic and control listeners (Hill et al., 1999). ${ }^{4}$ As noted above, these results are inconsistent with other reports of frequency discrimination deficits in samples of adults with dyslexia (Baldeweg et al., 1999; Hari et al., 1999; McAnally \& Stein, 1996). It is possible that procedural differences between studies may have contributed to the difference in outcome. For example, we used four-interval trials in our experiments, whereas McAnally and Stein and Hari et al. used three-interval and two-interval trials, respectively (cf. France et al., 2002). We also used a minimal-uncertainty procedure in which the standard frequency was constant throughout the experiments, whereas in McAnally and Stein's procedure (but not in that of Hari et al., 1999) there was a trial-by-trial rove in the standard frequency. Whether and why these and other procedural differences might have different consequences for dyslexic and control listeners will require further experimentation, but the differences in outcome suggest a role for nonsensory factors in accounting for group differences in this type of experiment.

As expected, frequency discrimination performance was affected by the addition of a masker for both groups of listeners, with an elevation in thresholds in the fixedmasker condition relative to those in the baseline nonmasker condition. There was an overall effect of masker type, with both masker variability (fixed vs. random) and spectral proximity of target and masker (different or same target-masker frequencies) affecting performance on this task for both the dyslexic and control listener groups. Threshold elevation relative to the baseline condition was smallest in the fixed-masker condition, consistent with a potential role for the masker as an additional cue to the target interval. However, the slightly greater threshold elevation in the short ISI condition suggests that the fixed masker may also have exerted a small masking effect working against the benefits afforded by a fixed-frequency referent. Threshold elevation was greater for random-frequency maskers

\footnotetext{
${ }^{4}$ Note that Hill et al. (1999) used 400-ms tones. Experiment 2 of the present study used 75-ms tones.
}

than for fixed maskers, consistent with the lack of a fixed-frequency referent in the random-masker conditions. Thresholds also were greater for the $1-\mathrm{kHz}$ random-masker condition than for the $2-\mathrm{kHz}$ randommasker condition, as predicted by Sparks (1976; but not by Bland \& Perrot, 1978).

A significant interaction between masker type and group was observed for the $2-\mathrm{kHz}$ random-frequency masker condition at 200-ms ISI, but the effect was not significant for the 20-ms ISI condition. These results suggest that, relative to control listeners, the frequency discrimination performance of the dyslexic participants was more adversely affected by the addition of a random-frequency masker. This pattern of performance was only observed reliably for mean thresholds when the target and masker were in different frequency regions, and was not specific to the short ISI condition. One possible interpretation of this finding is that the dyslexic listeners were less able to exploit the relative unmasking afforded when target and masker occupied different frequency regions. Consistent with this interpretation, post hoc tests revealed that the difference in thresholds between the two random-masker conditions was significant for the control group, but not for the dyslexia group. Moreover, the thresholds for the two groups did not differ in the 1-kHz random-masker condition when target and masker were in the same frequency region. We note that this result contrasts with Rosen and Manganari's (2001) finding that backward-masked detection thresholds for dyslexic listeners were significantly higher than those of controls only when the masker spectrally overlapped the signal. The greater similarity of mean thresholds in the $1-\mathrm{kHz}$ and $2-\mathrm{kHz}$ random-masker conditions for dyslexic listeners than for control listeners provides no support for the proposal that dyslexic listeners have an increased tendency to segregate tones of different frequency (Helenius et al., 1999). If this had been the case, then the difference between the $1-\mathrm{kHz}$ and $2-\mathrm{kHz}$ random-masker conditions should have been larger for dyslexic listeners than for control listeners.

Nonetheless, the absence of any reliable differences between dyslexic and control listeners in an analysis using the lowest of the four threshold estimates in each condition suggests that the single group difference should be interpreted cautiously. Inspection of the variance in thresholds across the four runs for individuals in both groups did not reveal the variability indexed learning or improvement in performance across runs (i.e., some individuals produced their "best" thresholds on the first run, others on the second or third).

When the distributions of frequency discrimination thresholds were examined, no statistical outliers were identified in the dyslexia group. This contrasts with the results reported by Hill et al. (1999), who noted a 
small subgroup of dyslexic listeners showing elevated frequency discrimination thresholds for relatively lowfrequency $(1 \mathrm{kHz})$ and high-frequency $(6 \mathrm{kHz})$ tones. In the present experiment, 1 outlier was identified in the control group. This individual was also an outlier in the TOD task, suggesting that his poor performance could have been due to poor frequency discrimination. A small number of listeners from both groups were classified as outliers in the masker conditions of the ABRM task. The individuals observed with impairments in the TOD task also were classified as outliers in the masker conditions of the ABRM task. In addition, a few listeners who had not experienced any difficulties on the TOD task had difficulties in the ABRM task. It is clear, therefore, that some individuals (both dyslexic and normal readers) experienced difficulty with these auditory tasks, but it is not clear that their difficulties indicate a genuine impairment in auditory processing. An alternative possibility is that, for reasons that might include limitations of memory or attention, some individuals may simply be poor at doing psychoacoustical tasks.

\section{Relation Between Auditory and Phonological Processing Skills}

The findings reported above indicate that, as a group, the dyslexic listeners did not differ significantly from control listeners on two measures of rapid auditory processing. However, our data appear consistent with Tallal's (1980) prediction that dyslexic individuals with the most severe phonological processing deficits are impaired on measures of rapid auditory processing. Moderate correlations were observed between the phonology and auditory measures using the data from the full sample, and correlational analyses performed using the separate data sets for the dyslexic and control groups provided some evidence for a weak association between phonological processing skill and performance on the TOD task using a short ISI. There was also an association between performance on the simple frequency discrimination task (ABRM-baseline) and phonological processing skill. In contrast, no evidence was found for an association between an individual's phonological processing skill and his or her ability to discriminate tone frequency when target tones were followed by masking tones.

Within the dyslexia group, those individuals whose performance levels were observed to fall consistently outside the normal range across the majority of the auditory tasks were also among those with the most severe phonological deficits, indexed by the phonology composite score. However, contrary to the hypothesized association, there were also participants with dyslexia who had weak phonological processing skills but relatively good levels of auditory processing abilities. In a similar vein, a subgroup of individuals from the control sample who were unimpaired in their literacy or phonological processing skills performed poorly on the auditory tasks. Such findings add to the accumulating evidence that auditory deficits are neither necessary nor sufficient to cause phonological difficulties (Bishop et al., 1999; Rosen \& Manganari, 2001). Furthermore, the observed associations between auditory processing and phonological processing skills do not speak to the direction of causality or to possible mediating factors. For example, common to both the auditory and phonological processing tasks is the need to allocate auditory attention.

Recent reviews have considered methodological factors that may explain the contradictory findings in the literature of studies examining auditory processing deficits in dyslexia (Bailey \& Snowling, 2002; Hirsh \& Watson, 1996; McArthur \& Bishop, 2001). The focus in the present study on high-achieving adults with dyslexia may have been a factor in our ability to find group differences in auditory processing. An aim of this study was to reduce the likelihood of including individuals with comorbid deficits such as SLI or ADHD, avoiding the introduction of an undesirable confound into the study (Ramus et al., 2003). However, we acknowledge the need for caution when generalizing these results to the wider dyslexia population.

In summary, we found no strong evidence in the present study to indicate that adults with dyslexia are subject to a rapid auditory processing deficit. Neither was there evidence for problems with the extraction of frequency information from sequences of nonverbal auditory tones separated by short ISIs. Moreover, the observation that a subgroup of participants, including both normal and dyslexic readers, had weak auditory processing skills weakens the argument for a causal relationship between auditory processing deficits and literacy impairments. Two possibilities remain open. First, auditory deficits may act as moderating variables (Holmbeck, 1977) and only have an impact on literacy development (when associated with a primary risk factor, such as genetic risk; Bishop et al., 1999). Second, auditory processing impairments may affect the creation of phonological representations early on in development (perhaps during the preschool years when they become segmentally organized). As development proceeds, auditory deficits may resolve sufficiently to be no longer detectable.

\section{Acknowledgments}

The work reported in this article was funded by Wellcome Trust Project Grant No. 55671. We thank the readers with dyslexia and the normal readers who took part. 


\section{References}

Ahissar, M., Protopapas, A., Reid, M., \& Merzenich, M. (2000). Auditory processing parallels reading abilities in adults. Proceedings of the National Academy of Sciences USA, 97, 6832-6837.

Au, A., \& Lovegrove, B. (2001). The role of visual and auditory temporal processing in reading irregular and nonsense words. Perception, 30, 1127-1142.

Bailey, P. J., \& Snowling, M. J. (2002). Auditory processing and the development of language and literacy. British Medical Bulletin, 63, 135-146.

Baldeweg, T., Richardson, A., \& Gruzelier, J. (1999). Impaired auditory frequency discrimination in dyslexia detected with mismatch evoked potentials. Annals of Neurology, 45, 495-503.

Bishop, D. V. M., Carlyon, R. P., Deeks, J. M., \& Bishop, S. J. (1999). Auditory temporal processing impairment: Neither necessary nor sufficient for causing language impairment in children. Journal of Speech, Language, and Hearing Research, 42, 1295-1310.

Bland, D. E., \& Perrot, D. R. (1978). Backward masking: Detection versus recognition. Journal of the Acoustical Society of America, 63, 1215-1217.

Cacace, A. T., McFarland, D. J., Ouimet, J. R., Schrieber, E. J., \& Marro, P. (2000). Temporal processing deficits in remediation-resistant reading-impaired children. Audiology and Neuro-Otology, 5(2), 83-97.

Castles, A., \& Coltheart, M. (1993). Varieties of developmental dyslexia. Cognition, 47, 149-180.

France, S. J., Rosner, B. S., Hansen, P. C., Calvin, C., Talcott, J. B., Richardson, A. J., \& Stein, J. F. (2002). Auditory frequency discrimination in adult developmental dyslexics. Perception \& Psychophysics, 64, 169-179.

Frederickson, N., Frith, U., \& Reason, R. (1997). Phonological Assessment Battery. Windsor, Berkshire, U.K.: NFER-Nelson.

Griffiths, Y. M., \& Snowling, M. J. (2002). Predictors of exception word and nonword reading in dyslexic children: The severity hypothesis. Journal of Educational Psychology, 94, 34-43.

Gross-Glenn, K., Jallad, B., Novoa, L., HelgrenLempesis, V., \& Lubs, H. A. (1990). Nonsense passage reading as a diagnostic aid in the study of adult familial dyslexia. Reading and Writing: An Interdisciplinary Journal, 2, 161-173.

Hari, R., Saaskilahti, A., Helenius, P., \& Uutela, K. (1999). Non-impaired auditory phase locking in dyslexic adults. Neuroreport, 10, 2347-2348.

Hatcher, J., Snowling, M. J., \& Griffiths, Y. M. (2002). Cognitive assessment of dyslexic students in higher education. British Journal of Educational Psychology, 72, 119-133.

Heath, S. M., Hogben, J. H., \& Clark, C. D. (1999). Auditory temporal processing in disabled readers with and without oral language delay. Journal of Child Psychology and Psychiatry, 40, 637-647.

Helenius, P., Uutela, K., \& Hari, R. (1999). Auditory stream segregation in dyslexic adults. Brain, 122, 907-913.
Hill, N. I., Bailey, P. J., Griffiths, Y. M., \& Snowling, M. J. (1999). Frequency acuity and binaural masking release in dyslexic listeners. Journal of the Acoustical Society of America, 106, L53-L58.

Hirsh, I. J., \& Watson, C. S. (1996). Auditory psychophysics and perception. Annual Review of Psychology, 47, 461-484.

Holmbeck, G. N. (1977). Toward terminological, conceptual and statistical clarity in the study of mediators and moderators: Examples from the child-clinical and paediatric psychology literatures. Journal of Consulting and Clinical Psychology, 65, 599-610.

Jastak, J., \& Wilkinson, G. J. (1993). Wide Range Achievement Test-Third Edition. Wilmington, DE: Jastak.

Kallman, H. J., \& Massaro, D. W. (1979). Similarity effects in backward recognition masking. Journal of Experimental Psychology: Human Perception and Performance, 5, 110-128.

Levitt, H. (1971). Transformed up-down methods in psychoacoustics. Journal of Acoustical Society of America, 49, 167-177.

Marshall, C. M., Snowling, M. J., \& Bailey, P. J. (2001). Rapid auditory processing and phonological ability in normal readers and readers with dyslexia. Journal of Speech, Language, and Hearing Research, 44, 925-940.

Massaro, D. W. (1970). Perceptual processes and forgetting in memory tasks. Psychological Review, 79, 124-145.

Massaro, D. W. (1972). Perceptual images, processing time and perceptual units in auditory perception. Psychological Review, 79, 124-145.

Massaro, D. W., \& Burke, D. (1991). Perceptual development and auditory backward recognition masking. Developmental Psychology, 27, 85-96.

McAnally, K. I., Castles, A., \& Stuart, G. W. (2000). Visual and auditory processing impairments in subtypes of developmental dyslexia: A discussion. Journal of Developmental and Physical Disabilities, 12(2), 145-156.

McAnally, K. I., \& Stein, J. F. (1996). Auditory temporal coding in dyslexia. Proceedings of the Royal Society of London, Series B, 263, 961-965.

McArthur, G. M., \& Bishop, D. V. M. (2001). Auditory perceptual processing in people with reading and oral language impairments: Current issues and recommendations. Dyslexia, 7, 150-170.

McArthur, G. M., \& Hogben, J. H. (2001). Auditory backward recognition masking in children with a specific language impairment and children with a specific reading disability. Journal of the Acoustical Society of America, 109, 1092-1100.

McArthur, G. M., Hogben, J. H., Edwards, V. T., Heath, S. M., \& Mengler, E. D. (2001). On the "specifics" of specific reading disability and specific language impairment. Journal of Child Psychology and Psychiatry, 41, 869-874.

Nittrouer, S. (1999). Do temporal processing deficits cause phonological processing problems? Journal of Speech, Language, and Hearing Research, 42, 925-942.

Ramus, F., Rosen, S., Dakin, S. C., Day, B. L., Castellote, J. M., White, S., \& Frith, U. (2003). Theories 
of developmental dyslexia: Insights from a multiple case study of dyslexic adults. Brain, 126, 1-25.

Reed, C. (1989). Speech perception and the discrimination of brief auditory cues in reading disabled children. Journal of Experimental Child Psychology, 48, 270-292.

Rosen, S., \& Manganari, E. (2001). Is there a relationship between speech and nonspeech auditory processing in children with dyslexia? Journal of Speech, Language, and Hearing Research, 44, 720-736.

Schulte-Korne, G., Deimel, W., Bartling, J., \& Remschmidt, H. (1998). Auditory processing and dyslexia: Evidence for a specific speech processing deficit. Neuroreport, 9, 337-340.

Sparks, D. W. (1976). Temporal recognition masking-or interference? Journal of the Acoustical Society of America, $60,1347-1353$

Studdert-Kennedy, M., \& Mody, M. (1995). Auditory temporal perception deficits in the reading-impaired: A critical review of the evidence. Psychonomic Bulletin \& Review, 2, 508-514.

Talcott, J. B., Witton, C., McClean, M., Hansen, P. C., Rees, A., Green, G. G. R., \& Stein, J. F. (1999). Can sensitivity to auditory frequency modulation predict children's phonological and reading skills? NeuroReport, 10, 2045-2050.

Tallal, P. (1980). Auditory temporal perception, phonics, and reading disabilities in children. Brain and Language, 9, 182-198.

Tallal, P., Allard, L., Miller, S., \& Curtiss, S. (1997). Academic outcomes of language impaired children. In C. Hulme \& M. Snowling (Eds.), Dyslexia: Biology, cognition \& intervention (pp. 167-181). London: Whurr.
Tallal, P., \& Piercy, M. (1973a). Deficits of nonverbal auditory processing in children with developmental aphasia. Nature, 241, 468-489.

Tallal, P., \& Piercy, M. (1973b). Developmental aphasia: Impaired rate of non-verbal processing as a function of sensory modality. Neuropsychologia, 11, 389-398.

Tallal, P., \& Stark, R. E. (1982). Perceptual/motor profiles of reading impaired children with or without concomitant oral language deficits. Annals of Dyslexia, 32, 163-176.

Torgeson, J. K., Wagner, R., \& Rashotte, C. (1999). Test of Word Reading Efficiency. Circle Pines, MN: American Guidance Service.

Waber, D. P., Weiler, M. D., Wolff, P. H., Bellinger, D., Marcus, D. J., Ariel, R., et al. (2001). Processing of rapid auditory stimuli in school-age children referred for evaluation of learning disorders. Child Development, 72(1), 37-49.

Wechsler, D. (1994). Wechsler Adult Intelligence ScaleRevised. San Antonio, TX: The Psychological Corporation.

Witton, C., Talcott, J. B., Hansen, P. C., Richardson, A. J., Griffiths, T. D., Rees, A., et al. (1998). Sensitivity to dynamic auditory and visual stimuli predicts nonword reading ability in both dyslexic and normal readers. Current Biology, 8, 791-797.

Received July 23, 2002

Accepted April 16, 2003

DOI: 10.1044/1092-4388(2003/105)

Contact author: Yvonne M. Griffiths, PhD, Department of Psychology, University of Essex, Colchester CO4 3SQ, United Kingdom. E-mail: ymg@essex.ac.uk 